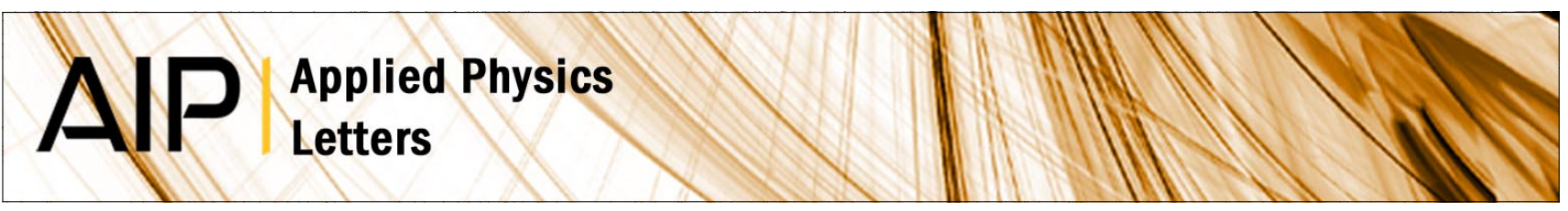

\title{
Zero-phonon emission and magnetic polaron parameters in EuTe
}

A. B. Henriques, G. D. Galgano, E. Abramof, B. Diaz, and P. H. O. Rappl

Citation: Appl. Phys. Lett. 99, 091906 (2011); doi: 10.1063/1.3634030

View online: http://dx.doi.org/10.1063/1.3634030

View Table of Contents: http://apl.aip.org/resource/1/APPLAB/v99/i9

Published by the American Institute of Physics.

\section{Related Articles}

Ambient molecular water accumulation on silica surfaces detected by a reflectance interference optical balance Appl. Phys. Lett. 97, 183702 (2010)

Blue-green light photochromism in europium doped BaMgSiO4

Appl. Phys. Lett. 97, 181905 (2010)

Spectral analysis of resonance ultrasonic spectroscopy: Kramers-Kronig analysis, Fano profiles, and the case of precursor softening in SnTe:Cr

Appl. Phys. Lett. 97, 111907 (2010)

Thermoluminescence properties of carbon doped Y3Al5O12 (YAG) crystal

J. Appl. Phys. 106, 033105 (2009)

Enhanced photochromism in nanostructured molybdenum trioxide films

Appl. Phys. Lett. 95, 051917 (2009)

\section{Additional information on Appl. Phys. Lett.}

Journal Homepage: http://apl.aip.org/

Journal Information: http://apl.aip.org/about/about_the_journal

Top downloads: http://apl.aip.org/features/most_downloaded

Information for Authors: http://apl.aip.org/authors

\section{ADVERTISEMENT}

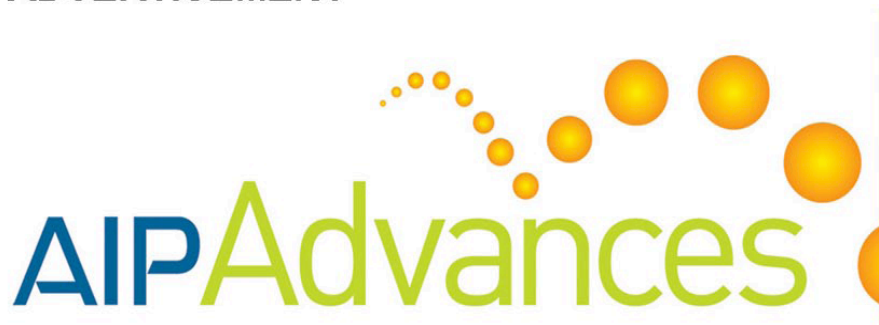

\section{Explore AIP's new}

open-access journal

Article-level metrics now available

Join the conversation!

Submit Now

Rate \& comment on articles 


\title{
Zero-phonon emission and magnetic polaron parameters in EuTe
}

\author{
A. B. Henriques, ${ }^{1, a)}$ G. D. Galgano, ${ }^{1}$ E. Abramof, ${ }^{2}$ B. Diaz, ${ }^{2}$ and P. H. O. Rappl ${ }^{2}$ \\ ${ }^{1}$ Instituto de Física, Universidade de São Paulo, C.P. 66318, 05315-970 São Paulo, Brazil \\ ${ }^{2}$ LAS-INPE, 12227-010 São José dos Campos, Brazil
}

(Received 28 June 2011; accepted 14 August 2011; published online 31 August 2011)

\begin{abstract}
A phonon structure in the photoluminescence of EuTe was discovered, with a well-defined zero-phonon emission line (ZPL). The ZPL redshifts linearly with the intensity of applied magnetic field, indicating spin relaxation of the photoexcited electron, and saturates at a lower magnetic field than the optical absorption bandgap, which is attributed to formation of magnetic polarons. From the difference in these saturation fields, the zero-field polaron binding energy and radius are estimated to be $43 \mathrm{meV}$ and 3.2 (in units of the EuTe lattice parameter), respectively. (C) 2011 American Institute of Physics. [doi:10.1063/1.3634030]
\end{abstract}

Optical control of magnetism is a topic of great current interest. ${ }^{1}$ Due to the large magnetic moment of an Eu atom $(S=7 / 2)$ and the high density of Eu atoms in an EuTe crystal with a transparency window in the visible/infrared, the control of magnetic order in EuTe could have applications in magneto-optical devices. The accepted EuTe level scheme consists of a localized $4 f^{7}$ valence state within the forbidden gap between the valence band and a $5 d\left(t_{2 g}\right)$ conduction band. This so-called $f d$ model describes very well the main magneto-optical properties, ${ }^{2,3}$ the quadratic dependence of the absorption bandgap on applied magnetic field, ${ }^{4}$ the emergence of narrow dichroic absorption lines in a magnetic field,$^{5-7}$ as well as non-linear optical properties of EuTe. ${ }^{8-11}$ However, photoluminescence (PL) observations suggest the activity of additional near-gap electronic energy levels beyond the $f d$ model. At high excitation intensities, a narrow PL emission band (width $10 \mathrm{meV}$ ) with a peak at $1.92 \mathrm{eV}$ is detected, which has been associated with an extra conduction band, described by a low density of states, lying below the $5 d\left(t_{2 g}\right)$ band. ${ }^{12}$

In this work, we report on the near-gap photoluminescence in EuTe, using much lower excitation power than in existing literature data. The samples were grown by molecular beam epitaxy on $\mathrm{BaF}_{2}$ substrates. ${ }^{13}$ The excitation source was a Nd:YAG $532 \mathrm{~nm}$ laser. Light was conveyed to the sample and collected from the sample using optical fibers. ${ }^{7}$ All measurements were taken at $4.8 \mathrm{~K}$. Typical PL spectra are shown as a function of excitation power in Fig. 1. At large excitation powers $\left(\sim 20 \mathrm{~W} / \mathrm{cm}^{2}\right)$, the narrow emission at $1.92 \mathrm{eV}$ is seen, in agreement with Ref. 14. However, when the excitation power is reduced by $4-5$ orders of magnitude $\left(\lesssim 2 \mathrm{~mW} / \mathrm{cm}^{2}\right)$, the PL develops an ensemble of equally spaced lines that combine into an emission band of total width $60 \mathrm{meV}$ with a maximum at $1.88 \mathrm{eV}$. This PL emission, henceforward labeled $\mathrm{MX}_{0}$-band, is the subject of the present investigation.

When a magnetic field, $B$, is applied (the Faraday geometry was used), the $\mathrm{MX}_{0}$ lines show a rigid giant redshift of $37 \mathrm{meV} / \mathrm{T}$, up to a saturation field, which is approximately

\footnotetext{
${ }^{\text {a) }}$ Author to whom correspondence should be addressed. Electronic mail: ahenriques@if.usp.br.
}

the same behavior reported for the $\mathrm{MX}_{1}$ line. ${ }^{12}$ However, in dramatic contrast to the $\mathrm{MX}_{1}$ emission, whose intensity decreases with $B$ and vanishes above $7.2 \mathrm{~T}^{12}$ at the highest field employed $(9.5 \mathrm{~T})$, the $\mathrm{MX}_{0}$ intensity remained more than $50 \%$ of the zero-field value. Fig. 2 shows the $\mathrm{MX}_{0}$ emission for samples of thickness $1,1.5$, and $4.2 \mu \mathrm{m}$, at $B=9.5$ $\mathrm{T}$. The spacing between the lines comprising the $\mathrm{MX}_{0}$ luminescence is independent of the thickness of the epitaxial layer, demonstrating that the line structure is not related to Fabry-Perot interference that causes modulation of the PL intensity of epitaxial EuTe grown on $\mathrm{BaF}_{2}$ substrates. ${ }^{12}$ The $\mathrm{MX}_{0}$ lines are better resolved in thicker layers because they arise from luminescing regions that are further away from the $\mathrm{BaF}_{2} / \mathrm{EuTe}$ interface, whose adjacency contains many structural defects due to the $6 \%$ lattice mismatch. ${ }^{13}$

For the thinnest $(1 \mu \mathrm{m})$ epitaxial sample, the FabryPerot modulation period of PL intensity ${ }^{12}$ is more than twice the $\mathrm{MX}_{0}$ linewidth; thus, it causes only a small effect on the $\mathrm{MX}_{0}$ lineshape. A Poisson distribution of intensities, $\frac{e^{-s} S^{N}}{N !}$, $N=0,1, \ldots$, fitted very well the $\mathrm{MX}_{0}$ lineshape for this

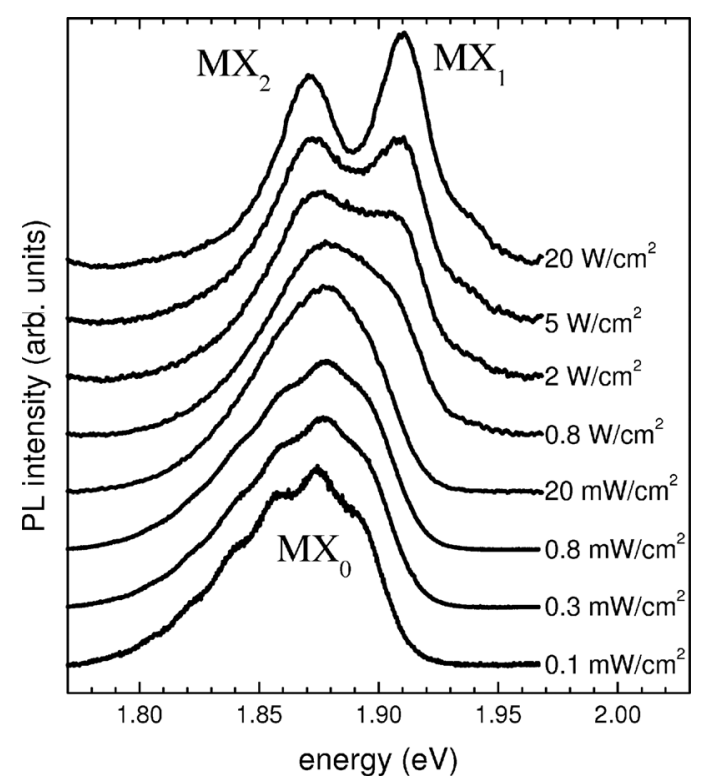

FIG. 1. PL spectra as a function of excitation power for an epitaxial EuTe layer of thickness $1.0 \mu \mathrm{m}$. 


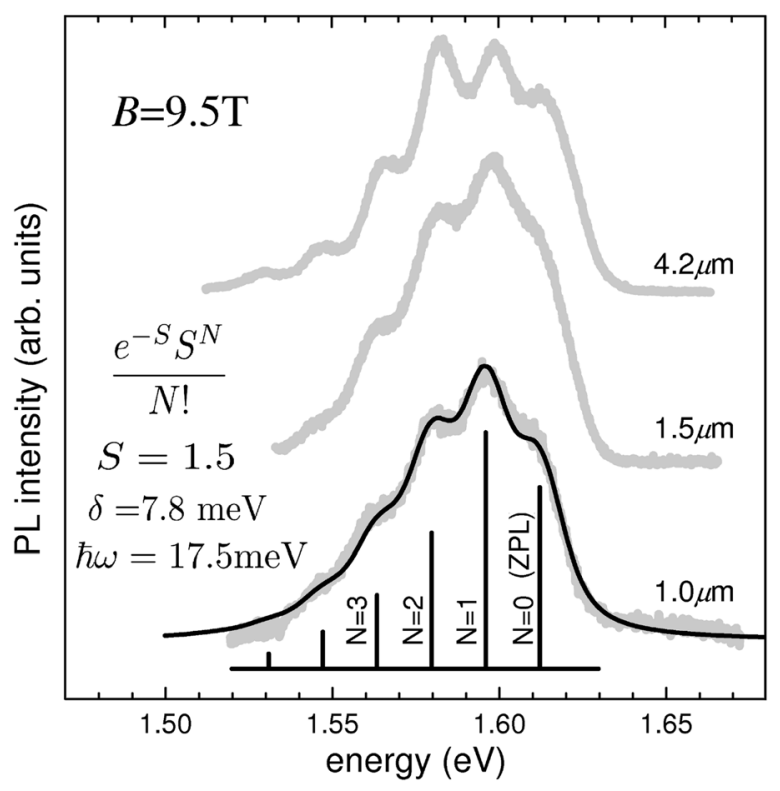

FIG. 2. PL spectra for excitation power $1 \mathrm{~mW} / \mathrm{cm}^{2}$.

sample at all fields, as shown by the thin black line in Fig. 2 for $B=9.5 \mathrm{~T}$, demonstrating that all $\mathrm{MX}_{0}$ lines arise from the same electronic transition coupled to a vibrational mode. ${ }^{15}$ The fitting yields the energy position of the zerophonon line as a function of $B$, the Huang-Rhys coupling strength $S=1.5$, the halfwidth of the individual lines $\delta=7.8$ $\mathrm{meV}$, and line spacing $\hbar \omega=17.5 \mathrm{meV}$. The latter matches exactly the energy of the LO-phonon in EuTe, ${ }^{16}$ which identifies the vibrational mode involved in the $\mathrm{MX}_{0}$ emission.

In order to interpret the magnetic field dependence of the ZPL energy, shown in Fig. 3, we first discuss briefly the absorption bandgap dependence on $B$, also displayed in Fig. 3 . The absorption dependence on the magnetic field is determined by the $d-f$ exchange interaction, ${ }^{4,17}$ characterized by an exchange integral, $J_{d f}$, between the electron in the excited $5 d\left(t_{2 g}\right)$ state and the magnetic-field-oriented $4 f^{7}$ lattice spins. Above the spin-flop field of $\sim 0.08 \mathrm{~T}$, the crystal contains a single domain described by two magnetic sublattices, oriented at an angle $\theta$ with respect to the magnetic field

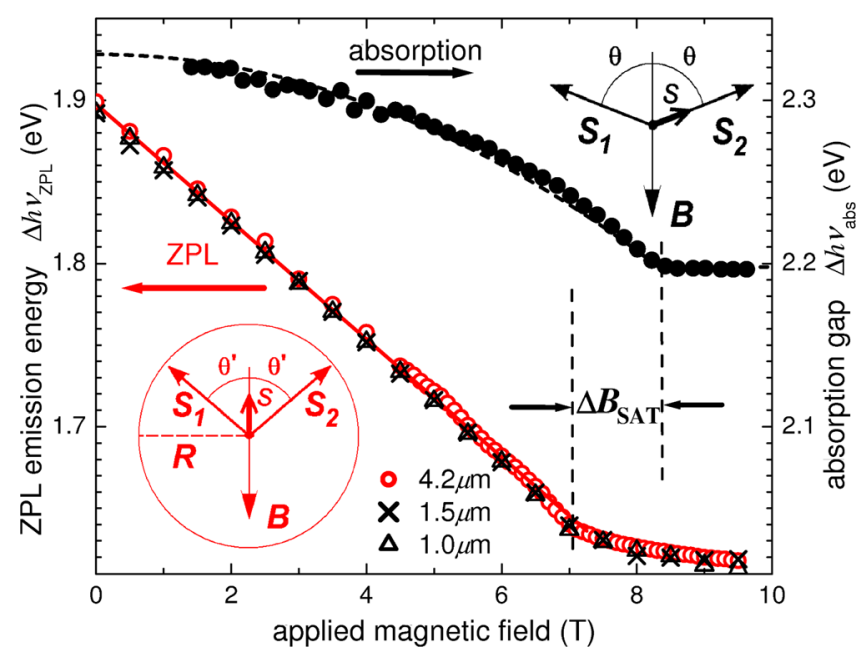

FIG. 3. (Color online) Absorption bandgap (from Ref. 5) and ZPL peak position for all samples as a function of $B$. direction as shown in the top right-hand side inset of Fig. $3 .^{7}$ Angle $\theta$ can be obtained by minimizing the molecular field energy of the $n$-th lattice spin in the fcc structure ${ }^{7,18}$

$$
E_{n}=-g_{S} \mu_{B} S B \cos \theta-6 J_{1} S^{2}-6\left(J_{1}+J_{2}\right) S^{2} \cos 2 \theta,
$$

thus,

$$
\cos \theta=\left\{\begin{array}{cl}
\frac{B}{B_{\mathrm{SAT}}} & \text { if } B<B_{\mathrm{SAT}} \\
1 & \text { if } B \geq B_{\mathrm{SAT}}
\end{array},\right.
$$

where $\quad B_{\mathrm{SAT}}=\frac{24\left|J_{1}+J_{2}\right| S}{g_{S} \mu_{B}}, \quad J_{1}=0.04 \pm 0.01 \quad \mathrm{~K} \quad$ and $J_{2}=-0.15 \pm 0.01 \mathrm{~K}$ are nearest neighbor and next-nearest neighbor exchange constants, ${ }^{2} g_{S}=2$, and $\mu_{B}$ is the Bohr magneton. Henceforward, we use the experimental value $B_{\mathrm{SAT}}=7.2 \mathrm{~T}$ (Ref. 19) (in the Faraday geometry used here, this internal field is achieved when the applied field is $8.3 \mathrm{~T}$, due to the demagnetization effect ${ }^{4}$ ). Due to spin conservation in the electric-dipole-allowed absorption process, immediately after excitation, the electron spin is oriented along the spin of a magnetic sublattice; moreover, following the Franck-Condon principle, the electronic transition takes place at fixed spatial and spin coordinates of the lattice; hence, the magnetic field dependence of the exchange energy of the absorbing state will be given by $\Delta h v_{a b s}=-\frac{1}{2} J_{d f} S(1+\cos 2 \theta)$, which according to Eq. (2) gives the quadratic dependence on $B$ shown by the dashed line in Fig. 3, in agreement with experiment. The maximum absorption redshift establishes $J_{d f} S=0.13 \mathrm{eV}$.

In contrast to the absorption line, the ZPL emission shows a linear dependence on $B$, and a saturation at a smaller magnetic field intensity (Fig. 3). Before recombination, the photoexcited electron relaxes exchange energy by flipping its spin towards a direction midway between the two sublattices, as shown schematically in the inset at the bottom lefthand side in Fig. 3. Therefore, the magnetic field dependent shift of the ZPL will be given by $\Delta h v_{Z P L}=-J_{X f} S \cos \theta$, which from Eq. (2) gives a linear dependence on $B$ as shown by the solid line in Fig. 3, in agreement with experiment. Here, another parameter $J_{X f}$ was introduced to characterize the exchange interaction between the relaxed photoexcited electron and the lattice spins. The value of $J_{X f} S$ is determined by the maximum redshift of the ZPL prior to saturation, giving $J_{X f} S \sim 0.27 \mathrm{eV}$.

The exchange interaction energy between a single $n$-th lattice spin and the relaxed photoexcited electron is equal to $\Delta_{X}=-\left|c_{n}\right|^{2} J_{X f} S \cos \theta$, where $\left|c_{n}\right|^{2}$ is the amplitude of the photoexcited electron wave function at the $n$-th lattice site. When a photoexcited electron is present this energy must be added to the right-hand side of Eq. (1). The additional term $\Delta_{X}$ favors the alignment of the lattice spins with the spin of the photoexcited electron, to form a magnetic polaron. ${ }^{17}$ Within a radius $R$ around the electron the tilt angle of the lattice spins is decreased to $\theta^{\prime}$, as shown schematically in the bottom left-hand side inset in Fig. 3. When $\Delta_{X}$ is introduced into Eq. (1), the saturation field decreases by $\Delta B_{\mathrm{SAT}}=\frac{J_{X f}}{g_{s} \mu_{B}}\left|c_{n}\right|^{2}$. If we approximate $\left|c_{n}\right|^{2}=\frac{1}{N},{ }^{17}$ where $N=\frac{4}{3} \pi R^{3} \frac{4}{a^{3}}$ is the number of Europium atoms within the polaron radius $R$ and $a=6.6 \AA$ is the EuTe lattice parameter, 


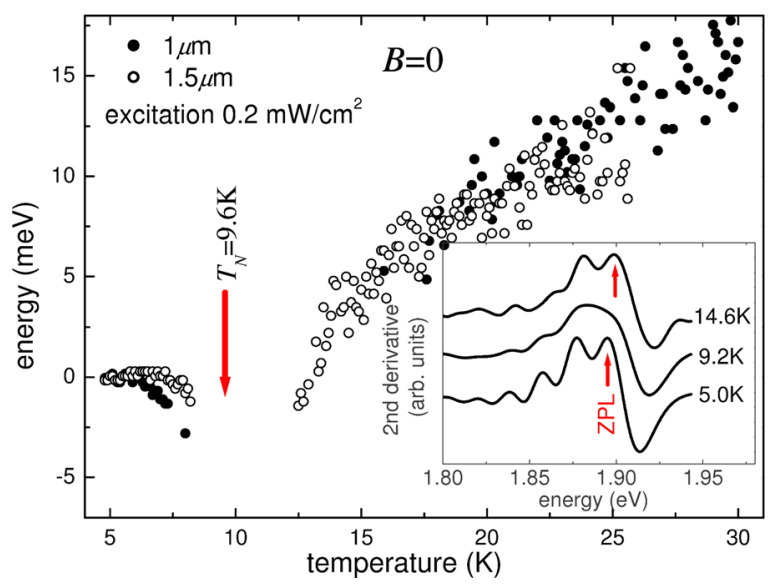

FIG. 4. (Color online) Temperature dependence of the ZPL energy, measured from its position at $\mathrm{T}=4.8 \mathrm{~K}$. The inset shows that the $\mathrm{ZPL}$ can be resolved in the second derivative of the PL signal at $5.0 \mathrm{~K}$ and $14.6 \mathrm{~K}$, but not at $9.2 \mathrm{~K}$.

then $\Delta B_{\text {SAT }}$ becomes a function of $J_{X f} S=0.27 \mathrm{eV}$ and $R$ only. Using the value $\Delta B_{\mathrm{SAT}}=1.2 \mathrm{~T}$ (see Fig. 3), the polaron radius is estimated to be $R \sim 3.2 a$.

The tilt angle $\theta_{0}$ of the lattice spins at $B=0$, within the magnetic polaron radius, is determined by $\cos \theta_{0}=\frac{\Delta B_{\mathrm{SAT}}}{B_{\mathrm{SAT}}}$, to give $\theta_{0} \sim 80^{\circ}$. Using $\theta_{0}$, the energy relaxation of the electron-lattice system due to the exchange interaction (the magnetic polaron binding energy, $E_{B}$ ) can be estimated, through $E_{B}=J_{X f} S \cos \theta_{0} \sim 43 \mathrm{meV}$. If we subtract $E_{B}$ from the Stokes shift of $430 \mathrm{meV}$ (see Fig. 3), the difference of $387 \mathrm{meV}$ is too large to be attributed to an electron-hole Coulomb binding energy. This is indicative that the $\mathrm{MX}_{0} \mathrm{lu}-$ minescence arises from the de-excitation of an electronic state lying below the $5 d\left(t_{2 g}\right)$ conduction band, as also observed for the $\mathrm{MX}_{1}$ luminescence. ${ }^{12}$ The absorbing and luminescing electronic states being different from one another also explains the numerical difference in the respective parameters $J_{d f} S$ and $J_{X f} S$.

In order to find independent support for our $E_{B}$ estimate, the temperature dependence of the ZPL emission was investigated, using an excitation power of only $0.2 \mathrm{~mW} / \mathrm{cm}^{2}$ and is shown in Fig. 4. Except for a narrow temperature interval around the Néel temperature, $T_{N}=9.6 \mathrm{~K}$, where the phonon lines broaden and cannot be resolved due to statistical fluctuations in the exchange field of the localized spins, the ZPL could be picked up in the second derivative of the PL up to about $30 \mathrm{~K}$, showing a blueshift of $\sim 20 \mathrm{meV}$. Above $T_{N}$, thermal spin fluctuations increasingly reduce the net magnetization within the polaron radius, thus reducing the depth of the exchange energy potential attracting the photoexcited electron. Theoretical modeling of magnetic polarons in EuTe indicate that the localization radius of the photoexcited electron remains nearly unaffected in the temperature range between $T_{N}$ and $10 T_{N}$ (Fig. 6 in Ref. 20), therefore mostly the exchange energy of the photoexcited electron changes in this temperature range. In this case the ZPL emission shift of $\sim 20 \mathrm{meV}$ between $T_{N}$ and $T=30 \mathrm{~K}$ establishes a lower bound for $E_{B}$, which is consistent with the value of $E_{B}=43$ meV deduced above. $E_{B}=43 \mathrm{meV}$ also agrees by order of magnitude with the value of $72 \mathrm{meV}$ reported by Heiss et $a l .,{ }^{12}$ which they deduced from an extrapolation of the Stokes shift of the $\mathrm{MX}_{1}$ line to infinitely high temperatures. Our estimate, however, has the advantage of being deduced from parameter $\Delta B_{\mathrm{SAT}}$ established accurately, obtained from a single temperature measurement, with no extrapolation required. The polaron type observed in this work, consisting of a conduction electron dressed by a halo of canted Eu spins, but without a ferromagnetic core, confirms the theoretical prediction of Mauger and Mills, ${ }^{21}$ who named such polarons as type-I. These results provide fundamental information on the basis of which to search for the optical control of magnetism in EuTe, using, for example, ultra-sensitive optical pump-probe techniques. ${ }^{22}$

This work was supported by Brazilian agencies CNPq and FAPESP. A.B.H. thanks V. Bindilatti for profitable discussions.

${ }^{1}$ J. Stohr and H. C. Siegmann, Magnetism: From Fundamentals to Nanoscale Dynamics (Springer, Berlin, 2006).

${ }^{2} \mathrm{P}$. Wachter, Handbook on the Physics and Chemistry of Rare Earths (North-Holland, Amsterdam, 1979), vol. 11, p. 507.

${ }^{3}$ A. Mauger and C. Godart, Phys. Rep. 141, 51 (1986).

${ }^{4}$ L. K. Hanamoto, A. B. Henriques, N. F. O., Jr., P. Rappl, E. Abramof, and Y. Ueta, J. Phys.: Condens. Matter 16, 5597 (2004).

${ }^{5}$ A. B. Henriques, A. Wierts, M. A. Manfrini, G. Springholz, P. H. O. Rappl, E. Abramof, and A. Y. Ueta, Phys. Rev. B 72, 155337 (2005).

${ }^{6}$ A. B. Henriques, M. A. Manfrini, P. H. O. Rappl, and E. Abramof, Phys. Rev. B 77, 035204 (2008).

${ }^{7}$ A. B. Henriques, G. D. Galgano, B. L. Diaz, P. H. O. Rappl, and E. Abramof, J. Phys.: Condens. Matter 19, 406234 (2007).

${ }^{8}$ B. Kaminski, M. Lafrentz, R. V. Pisarev, D. R. Yakovlev, V. V. Pavlov, V. A. Lukoshkin, A. B. Henriques, G. Springholz, G. Bauer, E. Abramof, P. H. O. Rappl, and M. Bayer, Phys. Rev. Lett 103, 057203 (2009).

${ }^{9}$ A. B. Henriques, E. Abramof, and P. H. O. Rappl, Phys. Rev. B 80 (2009).

${ }^{10}$ B. Kaminski, M. Lafrentz, R. V. Pisarev, D. R. Yakovlev, V. V. Pavlov, V. A. Lukoshkin, A. B. Henriques, G. Springholz, G. Bauer, E. Abramof, P. H. O. Rappl, and M. Bayer, Phys. Rev. B 81, 155201 (2010).

${ }^{11}$ M. Lafrentz, D. Brunne, B. Kaminski, V. V. Pavlov, A. B. Henriques, R. V. Pisarev, D. R. Yakovlev, G. Springholz, G. Bauer, E. Abramof, P. H. O. Rappl, and M. Bayer, Phys. Rev. B 82, 235206 (2010).

${ }^{12}$ W. Heiss, R. Kirchschlager, G. Springholz, Z. Chen, M. Debnath, and Y. Oka, Phys. Rev. B 70, 035209 (2004).

${ }^{13}$ B. Diaz, E. Granado, E. Abramof, P. H. O. Rappl, V. A. Chitta, and A. B. Henriques, Phys. Rev. B 78, 134423 (2008).

${ }^{14}$ W. Heiss, W. Prechtl, and G. Springholz, Appl. Phys. Lett. 78, 3484 (2001).

${ }^{15}$ A. B. Henriques and J. P. von der Weid, Solid State Commun. 56, 571 (1985).

${ }^{16}$ G. D. Holah, J. S. Webb, R. B. Dennis, and C. R. Pidgeon, Solid State Commun. 13, 209 (1973).

${ }^{17}$ T. Kasuya, A. Yanase, and T. Takeda, Solid State Commun. 8, 1543 (1970).

${ }^{18}$ J. Feinleib and C. R. Pidgeon, Phys. Rev. Lett. 23, 1392 (1969).

${ }^{19}$ N. F. O. Jr., S. Foner, and Y. Shapira, Phys. Rev. B 5, 2634 (1972).

${ }^{20}$ M. Umehara, Phys. Rev. B 52, 8140 (1995).

${ }^{21}$ A. Mauger and D. L. Mills, Phys. Rev. B 31, 8024 (1985).

${ }^{22}$ A. Schwan, B.-M. Meiners, A. B. Henriques, A. D. B. Maia, A. A. Quivy, S. Spatzek, S. Varwig, D. R. Yakovlev, and M. Bayer, Appl. Phys. Lett. 98, 233102 (2011). 